\title{
Prognostic Nomogram in Children and Adolescents with Central Nervous System Germ Cell Tumors
}

\section{Zetian Jia}

The Second Hospital of Hebei Medical University

\section{Yaqi Yan}

The First Hospital of Handan of Hebei Province

\section{Jiuxin Wang}

The Second Hospital of Hebei Medical University

He Yang

The Second Hospital of Hebei Medical University

Shuo Liu

The Second Hospital of Hebei Medical University

\section{Chengxi Han}

The Second Hospital of Hebei Medical University

Yuhua Hu ( $\sim$ huyuhua2000@163.com )

The Second Hospital of Hebei Medical University

\section{Research Article}

Keywords: germ cell tumors, SEER, nomogram, children

Posted Date: October 5th, 2021

DOl: https://doi.org/10.21203/rs.3.rs-934014/v1

License: (c) (1) This work is licensed under a Creative Commons Attribution 4.0 International License.

Read Full License 


\section{Abstract}

Background: The prognostic risk factors for primary central nervous system (CNS) germ cell tumors (GCTs) in children and adolescents remain controversial. This study aimed to develop and validate a nomogram that predicts 5-and 10-year survival rates for CNS GCTs in children and adolescents.

Methods: Pediatric with intracranial GCTs in the Surveillance, Epidemiology, and End Results (SEER) database from 2000 to 2018 were analyzed. All GCTs children were randomly divided into the development group (70\%) and the validation group (30\%). The least absolute shrinkage and selection operator (LASSO) was used to screen the characteristic variables, and the 5- and 10-year survival probability nomogram was constructed. The accuracy of the nomogram was evaluated by consistency index (C-index), calibration plot, receiver operating characteristic curve (ROC), decision curve analysis (DCA) and survival curve.

Results: We identified 819 cases of CNS GCTs in children. Three variables (histology, site and size) were selected by lasso regression to construct the nomogram. The $\mathrm{C}$-index of the development group and verification group is 0.744 and 0.759 . According to the time points of 5 and 10 years, the area under the curve of the development group is 0.753 and 0.696 , and the verification set is 0.787 and 0.718 . The results show that the prognostic nomogram developed by us has good accuracy.

Conclusions: This is the first prognostic nomogram of CNS GCTs in children and adolescents. It can effectively predict the 5- and 10-year survival probability of children. It provides a useful prediction tool for clinicians.

\section{Background}

Germ cell tumors (GCTs) originate from pluripotent embryonic germ cells during embryonic development and central nervous system (CNS) GCTs are one of the most common primary sites.[1, 2] CNS GCTs mostly occur in the deep part of the midline structures, and about $50 \%$ occur in the pineal gland.[3] Primary CNS GCTs are rare, with an incidence of approximately $0.1 / 100,000$ in the United States, accounting for less than $5 \%$ of all CNS tumors.[4] Intracranial CNS GCTs mainly occur in children and adolescent males and are almost all malignant tumors (except benign teratoma).[5] According to the histological features of intracranial GCTs, it can be divided into two categories: germinomatous GCTs (GGCTs) and nongerminomatous GCTs (NGGCTs).[3] The histomorphological characteristics of GGCTs are similar to those of spermatogonia of the testis in males or ovary dysgerminoma in females. In addition, heterogeneous NGGCTs are composed of a variety of mixed germ cell components (such as teratoma, choriocarcinoma and embryonal carcinoma, etc.), which have a very poor prognosis.[6]

The prognosis of GCTs is influenced by age, race, histological type, tumor location, tumor size, chemoradiotherapy, treatment and other risk factors.[7-9] Denyer et al. found in their study that germinoma patients with radiotherapy alone had a better prognosis than surgical resection.[8] In addition, the impact of different treatment methods on prognosis is also controversial. Unfortunately, there is no 
effective way to evaluate the prognosis of CNS GCTs in children and adolescents. Therefore, it is necessary to develop a clinically practical prediction model to predict the survival probability of germinoma of the central nervous system in children. The nomogram can use multivariate regression analysis, integrate multiple prediction indexes, and use line segments with scales to predict individual prognosis. It can also transform complex regression equations into visual images, making the results of predictive models more readable and easier to evaluate for patients.[10,11]

The Surveillance, Epidemiology and End Results (SEER) database is the largest clinical information collection platform in the United States, including demographic data, incidence rate, histology, treatment data and follow-up information. We conducted a prognostic study based on the SEER database in order to identify the characteristic variables that influence prognostic factors and predict the prognosis of intracranial GCTs in children and adolescents. In addition, we developed a nomogram model which can accurately predict the prognosis combined with characteristic variables and carried out internal validation.

\section{Methods}

\section{Study Population}

The SEER database is the largest cancer database in the United States, covering about one-third of the U.S. population. We obtained a license for the SEER*Stat database software for the analysis of clinical data (Username: 10901- November 2020). Data for clinical information on pediatric patients with intracranial germinoma were submitted in November 2020 from 18 registries. Criteria for inclusion included: (1) Age 0-19 years; (2) Diagnose GGCT or NGGCT; (3) The primary site is located in the intracranial; (4) Confirmed by microscopic histological examination. Patients with incomplete clinical information were excluded. The histological type of GGCTs was the International Classification of Diseases for Oncology, third edition (ICD-0-3) 9064 (Germinoma).[3] Histological ICD-0-3 numbers for NGGTs include 9065 (Germ cell tumor), 9070 (Embryonal carcinoma), 9071 (Yolk sac tumor), 9080 (Teratoma), 9082 (Malignant teratoma), 9085 (Mixed germ cell tumor), 9100 (Choriocarcinoma), and 9101 (Choriocarcinoma combined with other germ cell elements).[3] The primary site ICD-0-3 of the tumor is encoded as follows: C70.0-C70.9, C71.0-C71.9, C72.2-C72.5, C72.8-C72.9, C75.3 (Pineal gland), C75.1 (Pituitary gland), C75.2 (Craniopharyngeal duct).[2] 70\% of the children with germinoma meeting the criteria were randomly selected and assigned to the development group, and the rest were assigned to the validation group. Figure 1 shows the flow chart of screening children with intracranial germinomas from the SEER database in this study.

\section{Study design}

Clinical cancer patient statistics were collected for age, sex, and race at the time of diagnosis. Histological characteristics of cancer include histology, primary tumor site, tumor size, and surgery. Based 
on previous studies, the ages of children and adolescents were divided into 0-9 years old and 10-19 years old.[9] Demographic and racial information classification: white, black and other races. Acharya et al. classified germinoma into GGCTs and NGGCTs in their study.[3] The primary sites of tumors were the pineal gland, pituitary gland/ craniopharyngeal duct, and others. Germinoma sizes were less than $5 \mathrm{~cm}$ $(<5 \mathrm{~cm})$, greater than or equal to $5 \mathrm{~cm}(5 \mathrm{~cm}+)$, and unknown. Surgery includes surgical sets (codes 20,21 , $22,25,27,30,40,50,55,60,90$ ) and nonsurgical or unknown sets (codes 00,99$)$. Survival time for cancer patients is measured by the number of months of survival or the time of the last follow-up, and survival status includes alive or death.

\section{Development and validation of a nomogram}

Using the development-set data of children with germinoma obtained above, least absolute shrinkage and selection operator (LASSO) regression were used to screen the best predictive variable data.[12] These indexes were used to construct a nomogram model of 5- and 10-year survival probability of germinoma in children. The stability and accuracy of the nomogram model were verified by $\mathrm{C}$-index, calibration plot, receiver operating characteristic curve (ROC) and survival curve. In addition, the net benefit was predicted by the model is tested with decision curve analysis (DCA).[13]

\section{Statistical analysis}

The "caret" package was used to randomly select $70 \%$ of all patients as the model development population and $30 \%$ as the validation sample. Through "glmnet" and "survival" packages, LASSO regression was used to screen out the variables for constructing the nomogram, which was used to predict the 5- and 10-year survival probability of children germinoma. C-index is an assessment of how likely a model is to predict the patient's ability to have the event.[14] The calibration plot can be used to evaluate the accuracy of the model, which is the difference between the probability of the event predicted by the model and the actual event. In addition, the "rms", "foreign" and "survival" packages were used for internal validation of the model using bootstrapping technique on the basis of 1000 samples. The DCA curve of the multivariate COX regression model was plotted using the "ggDCA" package. The net benefit of the nomogram to children with germ cell tumor was evaluated the clinical practicability of the model at 5- and 10-year timepoint. The "survivalROC" package was used to draw the ROC curve of the 5- and 10year survival probability prediction model for children with germinoma. The prognostic index (PI) was constructed using the variables obtained from the above screening, and the best cut-off value was calculated. We divided PI into a high-risk group and low-risk group based on the best cutoff point and plotted the survival curve of the development group and verification group by "Kaplan-Meier" method. $\mathrm{P}<0.05$ was considered statistically significant. SEER ${ }^{\star}$ Stat software version 8.3.9

(https://seer.cancer.gov/seerstat/) was used to extract clinical data in patients with intracranial germ cell tumors. R software 4.0.5 (https://www.r-project.org) was used for statistical analysis and image production. 


\section{Results}

\section{Demographic characteristics}

A total of 819 germinoma patients who met the inclusion criteria were enrolled and divided into the training set (575 patients) and the validation set (244 patients). Most of the patients with intracranial germ cell tumors in the validation set and the test set were male children, accounting for $76.0 \%$ (437/575 patients) and $75.4 \%$ (184/244 patients) respectively. The histological diagnosis of GGCT was $73.4 \%$ (422/575 patients) in the development group and $73.0 \%$ (178/244 patients) in the validation group, respectively. The primary site of intracranial tumors was mainly in the pineal gland, with development set accounting for $46.1 \%$ (265/575 patients) and verification set accounting for $53.3 \%$ (130/244 patients). Univariate Cox regression analysis: age $(H R=0.81 ; 95 \% \mathrm{Cl}, 0.46$ to $1.43, \mathrm{P}=0.474)$, gender $(\mathrm{HR}=1.37$; $95 \% \mathrm{Cl}, 0.81$ to $2.31, \mathrm{P}=0.244)$, race $(\mathrm{HR}=0.96 ; 95 \% \mathrm{Cl}, 0.71$ to $1.30, \mathrm{P}=0.797)$, histological $(\mathrm{HR}=4.47$; $95 \% \mathrm{Cl}, 2.75$ to $7.25, \mathrm{P}<0.001)$, site $(\mathrm{HR}=1.25 ; 95 \% \mathrm{Cl}, 0.97$ to $1.62, \mathrm{P}=0.087)$, size $(\mathrm{HR}=1.72 ; 95 \% \mathrm{Cl}$, 1.30 to $2.28, P<0.001)$, surgery $(H R=0.82 ; 95 \% \mathrm{Cl}, 0.51$ to $1.33, \mathrm{P}=0.423)$. Table 1 summarizes the detailed demographic information and clinical histological characteristics of the cohort studies.

Table 1

Baseline characteristics of intracranial germ cell tumors in children from the SEER database. 
Characteristics

$\begin{array}{lll}\text { All patients } & \text { Training set } & \text { Validation } \\ \mathrm{N}=819(\%) & \mathrm{N}=575(\%) & \mathrm{N}=244(\%)\end{array}$

Age(years)

0.474

\begin{tabular}{|c|c|c|c|c|}
\hline $0-9$ & $188(23.0)$ & $131(22.8)$ & $57(23.4)$ & \\
\hline 10-19 & $631(77.0)$ & 444 (77.2) & $187(76.6)$ & \\
\hline Gender & & & & 0.244 \\
\hline Male & $621(75.8)$ & 437 (76.0) & 184 (75.4) & \\
\hline Female & 198 (24.2) & $138(24.0)$ & $60(24.6)$ & \\
\hline Race & & & & 0.797 \\
\hline White & 590 (72.0) & 395 (68.7) & 195 (79.9) & \\
\hline Black & 73 (8.9) & 57 (9.9) & $16(6.6)$ & \\
\hline Other & 156 (19.0) & 123 (21.4) & 33 (13.5) & \\
\hline Histological & & & & $P<0.001$ \\
\hline GGCTs & $600(73.3)$ & 422 (73.4) & 178 (73.0) & \\
\hline NGGCTs & 219 (26.7) & 153 (26.6) & 66 (27.0) & \\
\hline Site & & & & 0.087 \\
\hline Pineal gland & 395 (48.2) & $265(46.1)$ & 130 (53.3) & \\
\hline Pit/Cra gland & $73(8.9)$ & $52(9.0)$ & $21(8.6)$ & \\
\hline Other & 351 (42.9) & 258 (44.9) & $93(38.1)$ & \\
\hline Size (cm) & & & & $P<0.001$ \\
\hline$<5 \mathrm{~cm}$ & $369(45.1)$ & 264 (45.9) & 105 (43.0) & \\
\hline $5 \mathrm{~cm}+$ & 155 (18.9) & 107 (18.6) & 48 (19.7) & \\
\hline Unknown & $295(36.0)$ & 204 (35.5) & 91 (37.3) & \\
\hline Surgery & & & & 0.423 \\
\hline Yes & $411(50.2)$ & 279 (48.5) & $132(54.1)$ & \\
\hline No/Unknown & 408 (49.8) & 296 (51.5) & 112 (45.9) & \\
\hline
\end{tabular}

GGCTs: Germinomatous germ cell tumors; NGGCTs: Nongerminomatous germ cell tumors; Pit/Cra gland: Pituitary gland/ Craniopharyngeal duct 


\section{Feature selection and construction of the nomogram}

Data from children with an intracranial germinoma in the development group were used to screen out three characteristic variables as predictors of the construction of the prognostic nomogram by LASSO regression: histological (coefficient 1.20), site (coefficient 0.02), and size (coefficient 0.34). (Figure 2) A nomogram prognostic model was constructed based on LASSO regression results of the development data set. We used this prognostic model to predict 5- and 10-year survival rate in children with germ cell tumors. (Figure 3) The score of the nomogram is shown in Supplementary Table 1.

\section{Effect verification of nomogram}

We internally verified the model. The C-index of the development group was 0.744 , and that of the verification group was 0.759 . The 5 - and 10 -year survival prognostic models of intracranial germinoma in children showed good accuracy in the calibration plots in both the training cohort and the validation cohort. The net benefit of the predictive model for germinoma children was demonstrated by DCA. The Xaxis represents the risk threshold probability and the $\mathrm{Y}$-axis represents the clinical net benefit. As shown in Figure $4 \mathrm{C}$ and $\mathrm{D}$, our 5 - and 10-year survival probability prognostic models for the children with intracranial germinoma showed good net benefits.

\section{ROC and survival curve}

A multivariate COX regression model was established with germinoma histology, site and size as variables. The area under curve (AUC) values of the 5- and 10-year overall survival of the development group were 0.753 and 0.696 , and the AUC values of the validation set were 0.787 and 0.718 .(Figure $5 \mathrm{~A}$ and $\mathrm{B}$ ). The continuous variable $\mathrm{PI}$ is calculated using the three variables (histological, site, size). In the development set, the best cutoff point of $\mathrm{PI}$ is calculated with the 5- and 10-year survival time as the cutoff point is 0.85 . The cut-off point of 5 - and 10 -year survival time in the validation set was 1.27 . The population was divided into high-risk group and low-risk group according to the optimal cutoff point of the variable $\mathrm{PI}$ and the survival curve was plotted. Median follow-up time in children with intracranial germinoma development set was 92 months (1-227 months) and verification set was 80 months (1-227 months). The 5 -year survival rates were $96.5 \%$ and $80.9 \%$, and the 10 -year survival rates were $95.3 \%$ and $76.5 \%$, respectively, in the low-risk and high-risk children in the development set. The 5-year survival rates were $95.1 \%$ and $74.6 \%$, and the 10 -year survival rates were $92.6 \%$ and $70.5 \%$, respectively, in the low-risk and high-risk children in the validation group. Survival curve analysis showed that children with low-risk germinoma lived longer than those with high-risk germinoma in both the development and validation sets $(P<0.0001)$.

\section{Discussion}


In this retrospective cohort study based on SEER database, we developed and verified a new prognostic model based on three clinical variables (histology, site, size) for the first time. Compared with clinical risk factors, our model can improve the prediction ability of prognosis in children and adolescents with CNS GCTs.

Our results show that GCTs histology, tumor site and size may be important predictors of CNS GCTs survival in children and adolescents. In addition, we also established a nomogram based on high-risk factors to predict the 5- and 10-year survival probability of children CNS GCTs, and verified the stability of the model through internal validation.

Consistent with other cancers, the histological type of intracranial germinoma has a significant influence on the prognosis of children. In our study, it was found that NGGCTs scored 76 points in the nomogram constructed based on multivariate regression analysis, which was significantly higher than GGCTs. Compared with other germ cell tumors, NGGCTs have higher invasiveness and malignancy, and poor prognosis. This is consistent with the findings of Matsutani et al., that germinoma with different histological characteristics has a significant impact on the prognosis of patients.[15] In addition, the clinical treatment of NGGCTs generally requires the combination of surgery and postoperative radiotherapy and chemotherapy, and the treatment complications are also prognostic factors that cannot be ignored, which can be further explored in future studies.

In this study, the site of intracranial germinoma was only screened in LASSO regression but was not statistically significant in univariate COX regression $(P=0.087)$. This may be related to the incomplete statistics of the SEER database itself, as some tumors are present in multiple intracranial sites simultaneously. Intracranial germinoma usually occurs in the sellar region, pineal gland, basal ganglia and other parts. In our study, the number of germinomas in the pineal region was $48.2 \%$, and Acharya et al. also reported that about half of CNS GCTs occurred in the pineal region.[3] We found that tumor location was also an important factor affecting the prognosis of children. This may be due to the fact that the pineal region is located in an important intracranial anatomical site, which may cause increased intracranial pressure, diabetes insipidus, sleepiness and other changes in consciousness. In addition, because the pineal region is located deep in the brain, it is difficult to treat, resulting in a poor prognosis of tumors in this region.

Tumor size is known to be an important prognostic factor in many solid tumors. The variables screened by us through LASSO also included tumor size, indicating that the prognosis of CNS GCTs was significantly correlated with it. We found that the poor prognosis of CNS GCTs in children and adolescents was associated with tumor size, and the prognosis was worse as the number of tumors increased. This may be associated with a large germ cell tumor infiltrating the surrounding tissue and requiring craniotomy and postoperative chemoradiotherapy. And the treatment of small tumors jin only simple chemoradiotherapy. It is worth noting that craniotomy itself can cause great collateral damage to children, especially deep brain tumors. Therefore, in future studies, the size of the tumor needs to be further refined. Unlike previous studies, we did not find a correlation between age, sex, race, or treatment 
and the outcome of intracranial germinoma in children. This may be caused by a difference in the way we classify variables.

Currently, the impact of risk factors related to CNS GCTs on prognosis remains controversial. In addition, independent prognostic factors may be difficult to predict the prognosis of germinoma. A variable PI was calculated using the three risk factors, and the children were classified into high-risk and low-risk groups. The survival curve (Fig. 5, C and D) showed that there was a significant difference in survival time between the high-risk group and the low-risk group, and the difference between the development group and the validation group was statistically significant $(P<0.0001)$. We tested the stability and accuracy of the new prognostic nomogram model. The consistency index of the model in the development group and the validation group was 0.744 and 0.759 , indicating that the model has the good predictive ability and can be used in clinical practice. In summary, the prediction model constructed by multiple factors has a more significant effect on prognosis than that of a single factor, and it is a very promising method for predicting prognosis in the future.

There are still limitations to the study. First, the SEER database is retrospective data with limited clinical information of patients, and there may be selection bias in inpatient data. Future randomized controlled trials are needed to validate prognostic risk factors for germinoma. Second, the current prognostic model is only validated based on internal data, and external validation is needed in the future. Nevertheless, our nomogram helps predict the prognosis of CNS germinoma in children with good accuracy.

\section{Conclusion}

To our knowledge, this is the first study to develop a prognostic model for CNS GCTs in children and adolescents based on large sample size. Our results suggest that the nomogram can effectively predict 5and 10-year survival probability in children and adolescents with CNS GCTs, thus providing clinicians with a useful prognostic tool.

\section{Abbreviations}

GCTs: germ cell tumors; CNS: central nervous system; GGCTs: germinomatous germ cell tumors; NGGCTs: nongerminomatous germ cell tumors; SEER: Surveillance, Epidemiology and End Results; LASSO: least absolute shrinkage and selection operator; ROC: operating characteristic curve; DCA: decision curve analysis; PI: prognostic index;

\section{Declarations}

\section{Acknowledgements}

We are very grateful to the SEER program for approving the registration, and to the SEER database. 


\section{Funding}

Not applicable.

\section{Data availability statement}

Retrieved from the SEER* STAT database according to the SEER research data usage protocol.

\section{Ethical approval statement}

Not applicable.

\section{Consent for publication}

Not applicable.

\section{Competing interests}

The authors declare that they have no competing interests.

\section{Author contribution statement}

ZJ: Study design, formal analysis, visualization, manuscript writing; YY: Study design, data administration, review and editing; JW, HY, SL and CH: Data curation, methodology, validation and review and editing; $\mathrm{YH}$ : Supervision, investigation, review and editing.

\section{References}

1. Chen KS, Fustino NJ, Shukla AA, Stroup EK, Budhipramono A, Ateek C, Stuart SH, Yamaguchi K, Kapur P, Frazier AL et al: EGF Receptor and mTORC1 Are Novel Therapeutic Targets in Nonseminomatous Germ Cell Tumors. Mol Cancer Ther 2018, 17(5):1079-1089.

2. Villano JL, Virk IY, Ramirez V, Propp JM, Engelhard HH, McCarthy BJ: Descriptive epidemiology of central nervous system germ cell tumors: nonpineal analysis. Neuro-oncology 2010, 12(3):257-264.

3. Acharya S, DeWees T, Shinohara ET, Perkins SM: Long-term outcomes and late effects for childhood and young adulthood intracranial germinomas. Neuro-oncology 2015, 17(5):741-746.

4. Modak S, Gardner S, Dunkel IJ, Balmaceda C, Rosenblum MK, Miller DC, Halpern S, Finlay JL: Thiotepa-based high-dose chemotherapy with autologous stem-cell rescue in patients with recurrent 
or progressive CNS germ cell tumors. Journal of clinical oncology: official journal of the American Society of Clinical Oncology 2004, 22(10):1934-1943.

5. Poynter JN, Fonstad R, Tolar J, Spector LG, Ross JA: Incidence of intracranial germ cell tumors by race in the United States, 1992-2010. Journal of neuro-oncology 2014, 120(2):381-388.

6. Louis DN, Ohgaki H, Wiestler OD, Cavenee WK, Burger PC, Jouvet A, Scheithauer BW, Kleihues P: The 2007 WHO classification of tumours of the central nervous system. Acta Neuropathol 2007, 114(2):97-109.

7. Poynter JN, Amatruda JF, Ross JA: Trends in incidence and survival of pediatric and adolescent patients with germ cell tumors in the United States, 1975 to 2006. Cancer 2010, 116(20):4882-4891.

8. Denyer S, Bhimani AD, Patil SN, Mudreac A, Behbahani M, Mehta Al: Treatment and survival of primary intracranial germ cell tumors: a population-based study using SEER database. J Cancer Res Clin Oncol 2020, 146(3):671-685.

9. Williams LA, Frazier AL, Poynter JN: Survival differences by race/ethnicity among children and adolescents diagnosed with germ cell tumors. International journal of cancer 2020, 146(9):24332441.

10. lasonos A, Schrag D, Raj GV, Panageas KS: How to build and interpret a nomogram for cancer prognosis. Journal of clinical oncology: official journal of the American Society of Clinical Oncology 2008, 26(8):1364-1370.

11. Jia Z, Yan Y, Wang J, Yang H, Zhan H, Chen Q, He Y, Huang C, Hu Y: Development and validation of prognostic nomogram in ependymoma: A retrospective analysis of the SEER database. Cancer Med 2021.

12. Sauerbrei W, Royston P, Binder H: Selection of important variables and determination of functional form for continuous predictors in multivariable model building. Stat Med 2007, 26(30):5512-5528.

13. Vickers AJ, Elkin EB: Decision curve analysis: a novel method for evaluating prediction models. Med Decis Making 2006, 26(6):565-574.

14. Harrell FE, Jr., Lee KL, Mark DB: Multivariable prognostic models: issues in developing models, evaluating assumptions and adequacy, and measuring and reducing errors. Stat Med 1996, 15(4):361-387.

15. Matsutani M, Sano K, Takakura K, Fujimaki T, Nakamura O, Funata N, Seto T: Primary intracranial germ cell tumors: a clinical analysis of 153 histologically verified cases. $J$ Neurosurg 1997 , 86(3):446-455.

\section{Figures}


Patients with germ cell tumors in

Surveillance Epidemiology and End

Results 18 reg istry database $(2000-2018)$

\begin{tabular}{|l|}
\multicolumn{1}{|c|}{ Inclusion criteria } \\
(1) Age: 0-19 years \\
(2) ICD-O-3 histo logy co des: GGCTs (9064), NGGCTs (9080 \\
9085, 9101, 9100, 9065, 9070, 9082, and 9071) \\
(3) ICD-O-3 site co des: Pineal g land (C75.3), Pituitary g land/ \\
Craniopharyng eal duct (C75.1, C75.2); Other (C70.0-C70.9, \\
C71.0-C71.9, C72.2-C72.5, C72.8-C72.9) \\
(4) Microscopically confirmed
\end{tabular}

Elig ible children with germ cell tumors $(\mathrm{n}=825)$

\section{Excluded}

(1) The follow-up time is unknown $(n=6)$;

(2) Incomplete information $(n=0)$.

Patients for analys is $(\mathrm{n}=819)$;

Randomly divided into development set $(\mathrm{n}=575)$ and validation set $(\mathrm{n}=244)$.

\section{Figure 1}

Flowchart of participant inclusion and exclusion
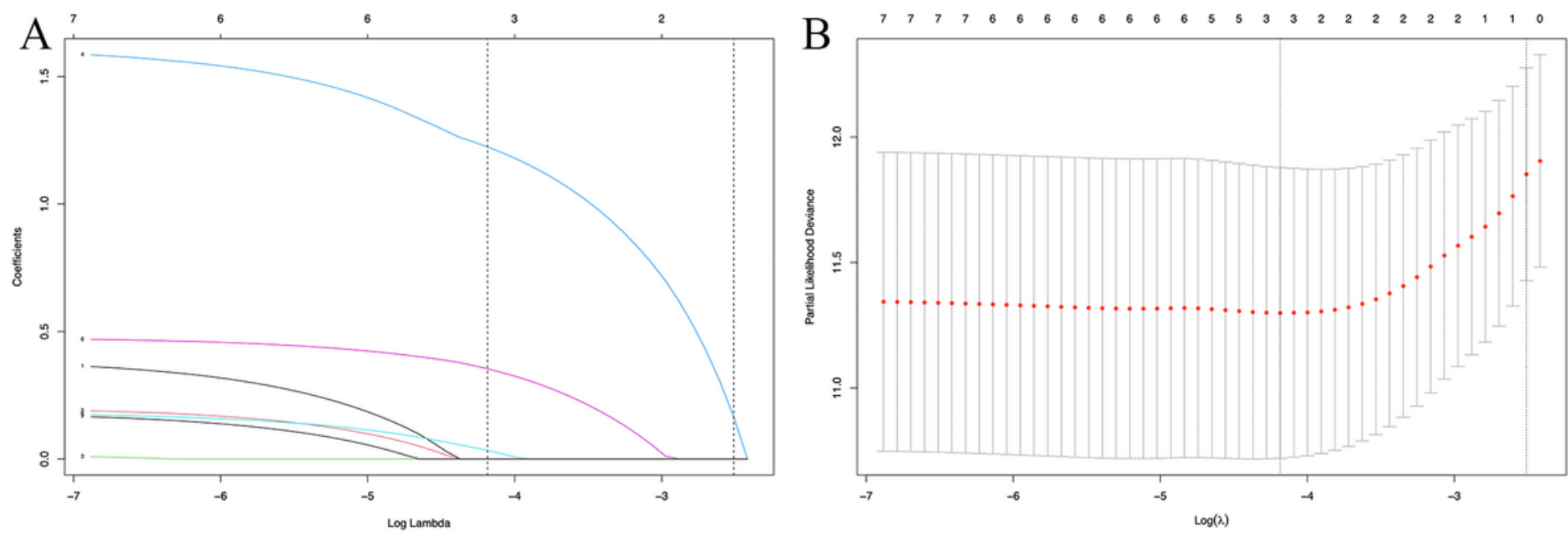
Figure 2

LASSO regression model was used to select characteristic impact factors. (A) LASSO coefficients of 3 features; (B) Selection of tuning parameter $(\lambda)$ for LASSO model

Points

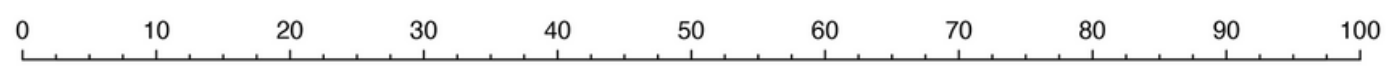

Site

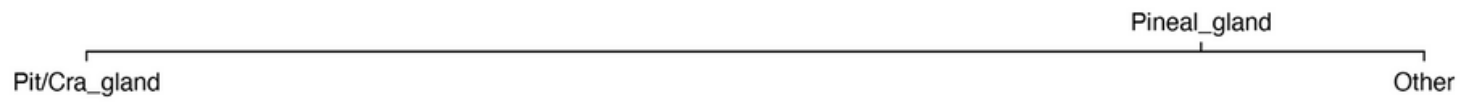

Histological

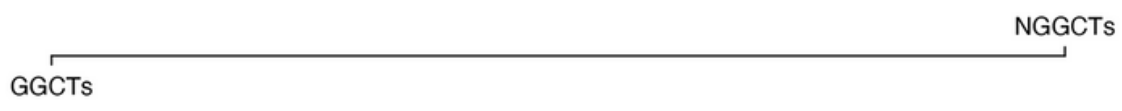

Size

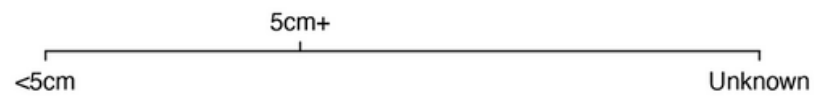

Total Points

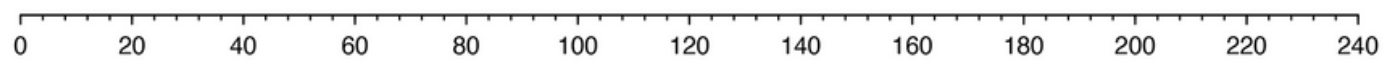

5-years Survival Probability

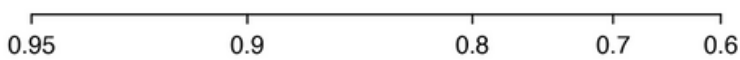

10-years Survival Probability

\begin{tabular}{llllll}
\hline 0.95 & 0.9 & \multicolumn{1}{c}{} & & \\
\hline & 0.8 & 0.7 & 0.6 & 0.5
\end{tabular}

\section{Figure 3}

Nomograms predicting 5- and 10-year survival probability. Pit/Cra gland: Pituitary gland/ Craniopharyngeal duct; GGCTs: Germinomatous germ cell tumors; NGGCTs: Nongerminomatous germ cell tumors 

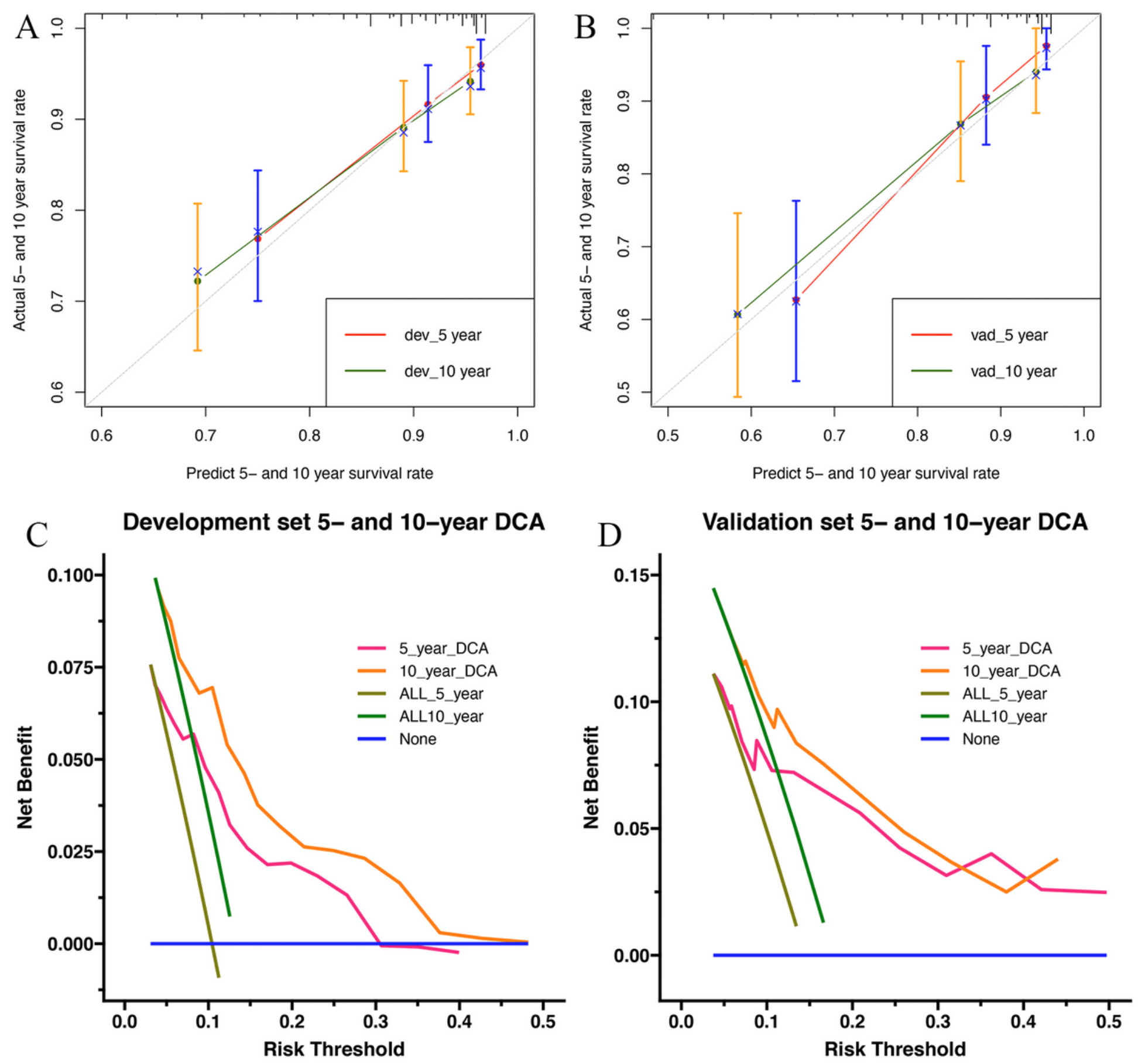

Figure 4

(A) 5- and 10-year calibration plot of the development group; (B) 5- and 10-year calibration plot of the validation group; (C) 5- and 10-year DCA of the development group; (D) 5- and 10-year DCA of the validation group. 

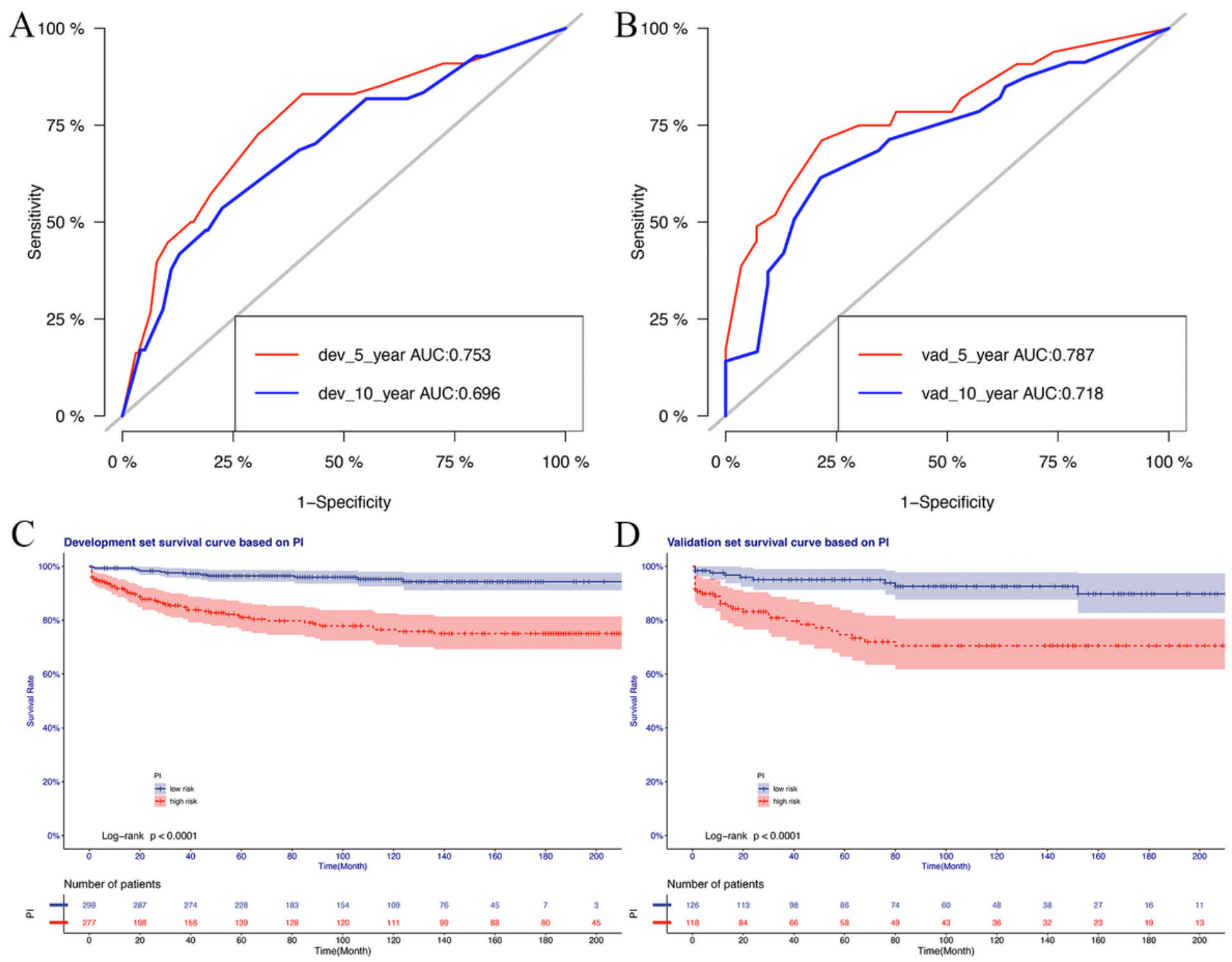

\section{Figure 5}

(A) 5- and 10-year ROC of the development group; (B) 5- and 10-year ROC of the validation group (C) Survival curve of development group based on prognosis index; (D) Survival curve of validation group based on prognosis index

\section{Supplementary Files}

This is a list of supplementary files associated with this preprint. Click to download.

- Additionalfile1.docx

- Additionalfile2.csv

- Additionalfile3.csv 